\title{
The Effect of the Calcium Antagonist Nifedipine on Pressor and Aldosterone Responses to Angiotensin II in Normal Man
}

\author{
J. A. Millar*, K. A. McLean' ${ }^{1}$ D. J. Sumner ${ }^{2}$, and J. L. Reid ${ }^{1}$ \\ 'University of Glasgow, Department of Materia Medica and 'Department of Clinical Physics and Bio-Engineering, \\ Stobhill General Hospital, Glasgow, UK
}

Eur J Clin Pharmacol (1983) 24: 315-321

In the above-mentioned paper the legends for Figs. 5

and 6 have been transposed.

\section{Announcements}

\section{Drug Monitoring}

Wien, October 22, 1983

The Austrian Working Group for Clinical Pharmacology announces this symposium.

For further information please contact:

Dr. H. Rameis

I. Med. Univ. Klinik Wien

Lazarettgasse 14

A-1090 Wien

\section{Biological Rhythms and Medications}

First International Montreux Conference

March 26-30, 1984

For further information please contact:

Chronopharmacology-Montreux

P.O. Box 32366

San Antonio, Texas 78216, USA

\section{Clinical Pharmacology}

International League Against Epilepsy

1983 Award

The Fourth Commission on Antiepileptic Drugs of the International League Against Epilepsy institutes a yearly award in Clinical Pharmacology.

The award is intended to recognize significant contributions, to the clinical pharmacology of antiepileptic drugs. Candidates may apply directly or be nominated. Each submission should include: Curriculum vitae, complete bibliography and 6 copies of the most significant publications.

The deadline for submission is December 31, 1983. All material should be forwarded to:

Lennart Gram, M.D.

Secretary of the

Commission on Antiepileptic Drugs

Department of Neurology

Bispebjerg Hospital

DK-2400 Copenhagen NV

Denmark 